COMMISSION No. 35

\title{
STELLAR CONSTITUTION (CONSTITUTION DES ETOILES)
}

\author{
Report of Meetings: 5 and 6 August 1988
}

PRESIDENT: D. Sugimoto

SECRETARY: A. Maeder and J. C. Wheeler

5 August 1988

\section{BUSINESS MEETING}

It was started by a moment of silence with the members standing in respect for a member, Max Krook, that had passed away since the last meeting in 1985.

New officers were voted for in an election held in 1987, and the results were reported by the President. The President, Vice-President and the organizing Committee members for 1988-91 were endorsed during the General Assembly. The new President is Andre Maeder (Switzerland) and the new Vice-President is Pierre Demarque (USA). During the last triennium the number of organizing Committee members exceeded the usual number by one, but it was corrected at this meeting. The new Organizing Committee consists of the six carryover people and the three new ones: D.O. Gough (UK), I. Iben, Jr. (USA), R. Kippenhahn (FRG), K. Nomoto (Japan), Y. Osaki (Japan), D. Sugimoto (Japan), J.W. Truran (USA), A.V. Tutukov (USSR), and J.C. Wheeler (USA).

From the membership list four names were deleted; one for the deceased, another for having left from astronomy, and the two others for resignation from commission 35 because their interests are now in other topics of astronomy. Twenty new members were accepted of which five had been the members of the Union and fifteen were new members accepted at this General Assembly. The total membership is now 308 . This implies that the interest is being kept at high level in this field of stellar Constitution.

One of the members proposed that Commission 35 should recommend an astronomer from a country for a new membership of the Union. There was much discussion, because the National committe of his country had just forgotten to observe the deadline to propse it to the Union, and because the proposed astronomer was as yet a Ph.D. candidate. However, considering the importance of getting into communication with countries having a small number of astronomers, commission 35 accepted it and proposed him for the membership of the Union.

Four proposed IAU sponsored meetings were discussed. Their titles were 1) Physics of Classical Novae, 2) Inside the Sun, 3) Rotation and Angular Momentum Evolution of Low-Mass Stars, 4) The Sun and cool Stars; activity, magnetism and dynamos, and 5) Evolution of Stars; the photospheric abundance connection. They were considered to be convened in 1989 or 1990. Some of them got enthusiastic support, which had already or have been recommended to the Exective committee. others got some comments concerning the scope of the topics which are now being taken into consideration by the proposers.

During the last triennium four circular letters were mailed to all members of Commission 35. One of the members expressed his opinion that communication among the members was nevertheless not enough, that the Commission should set up a research project to concentrate the coming triennium in specified topics, and that the organizing Committee should meet somewhere to secure it at least once during the 
triennium. However, considering that Commission 35 is oriented more to theoretical than observational researches, it was stressed as more important to exchange free ideas than to concentrate in specified topics. In this relation use of electronic mail was strongly encouraged and the Astronomical E-Mail Directory, that was prepared and being expanded by Dr. Chris Benn at the Royal Greenwich Observatory, will be most helpful.

\section{SCIENTIFIC MEETINGS}

There were six scientific meetings of Commission 35; two concerned directly with its main topics of stellar structure and evolution, three as the Joint Commission Meetings with Commissions 10, 12, 29, 36, and 44 on solar and stellar coronae, with 29, 36, 37, and 47 on spectroscopy of individual stars in globular clusters and the early chemical evolution of the galaxy, and with 28 and 37 on star clusters in the Magellanic Clouds, and the last one as a brainstorming session on Early type pulsating stars. Only the first two of these meetings are reported in what follows. The Joint Commission Meetings are reported elsewhere. The brainstorming session was organized by $M$. Aizenman but no report will be given here because of its informal nature.

Commission 35 also supported two Joint Discussions: Formation and evolution of stars in binary systems, and supernova $1987 \mathrm{~A}$ in the Large Magellanic Cloud. Their Proceedings will be found in Hilights of Astronomy.

\section{August 1988}

\section{Final stages of stellar Evolution}

Chaired by J. Craig Wheeler

J. Liebert Luminosity Function of White Dwarfs

E. Robinson Observational Constraints on Binary White Dwarfs

I. Iben, Jr. Theory of Binary White Dwarfs

z. Barkat Carbon Ignition in White Dwarfs

With the attention SN 1987A has brought to massive stars, the topic of a special Joint Discussion, the decision was made to focus this Comission meeting on white dwarfs. The study of white dwarfs has been one of the most exciting topics in the field of stellar evolution in the last few years yielding new insights into binary evolution, supernovae, and cosmology. The program was chosen to reflect these developments.

Liebert of the University of Arizona discussed the determination and implication of the luminosity function of white dwarfs. White dwarfs are not observed at magnitude limits beyond which fainter main sequence dwarfs are still detected. This implies a real cutoff in the white dwarf luminosity function. Theoretical cooling curves suggest that white dwarfs should live longer than a Hubble time and hence the paucity of dim white dwarfs sets a limit to the age of the white dwarfs. This age, presumably the age of the Galactic disk is about 9 billion years, a very low number by most expectations. Liebert reported that improved parallaxes have moved some stars to lower I and reduced this figure to about 8 billion years. Comments from the audience suggested that re-examination of opacities, etc., have resulted in little change in independent cooling calculations, with the exception of the effects of crystallization if it comes in at just the right epoch. Liebert remarked that some white dwarfs may be missing, hidden by binary companions, by having small tangential velocities or by scale height inflection, reducing the local space density, but none of these seem adequate to shift the observed downturn in the luminosity function. Liebert finally pointed out that a small sample of white 
dwarfs with high proper motion signifying halo stars may represent a first step toward a luminosity function for Population II stars.

Robinson of the University of Texas summarized the situation regarding the incidence of binary white dwarfs. Naive estimates suggest that as many as 1 in 10 white dwarfs should be in a close binary system. This is an important question because such systems can be an important source of gravitational radiation background, supernova progenitors, and testing grounds for theories of binary evolution. of 13 published white dwarf pairs (plus one or two added from the audience), 6 are visual binaries of large separation evolving as separate stars, 1 is astrometric, 2 are spectroscopic, still with periods too long to be subject to gravitational radiation, and only four are interacting. Among the latter, none are candidates for supernova explosions because of the low total mass. Several surveys have been conducted for binary white dwarfs. Spectral classification schemes have found four, but the technique is subject to strong selection effects, making it less systematic. Radial velocity surveys have turned up 1-gandidate ${ }_{3}$ in 67 systems surveyed. For a local supernova rate of $(4 \pm 2.5) \times 10^{-4} \mathrm{yr}^{-1} \mathrm{pc}$ the required density of binary white dwarfs with orbital period shorter than 3 hrs is $(1.6 \pm 1) \times 10^{-5 c}$. Robinson and Shafter set an observational limit on the total number of binary white dwarfs $3 \times 10^{-5} \mathrm{pc}^{-3}$ of which only about $1 / 10$ could be supernova progenitors. Thus the derived upper limit is two to eight times less than the required rate for SN Ia progenitors.

There are several problems that need to be studied further. One is the rate of supernova which is still strongly subject to small number statistics. The work of Robinson and shafter only specifically constrains systems born with orbital period in excess of 3 hours. If they are born at ultrashort periods, the current constraints may not apply. Finaliy Robinson cautioned that a selectively high proportion of high mass white dwarfs could undergo gravitational settling to become DC white dwarfs. The lack of lines in these systems would render them invisible to any radial velocity search technique. Thus it remains possible that SN Ia arise selectively from massive DC-DC white dwarf pairs.

Iben of the University of Illinois discussed current theoretical ideas on the origin and evolution of binary white dwarfs. He cited GP Com as an established He-He pair and $\mathrm{Hz} 29$ as a possible CO-He pair. He also argued that the sdo and sdB stars could arise in the fusing of a $\mathrm{CO}$ and $\mathrm{a}$ He dwarf and hence be indirect evidence for binary white dwarf evolution. Related arguments suggest an origin of white dwarfs like 40 Eri $B$ with only 0.43 solar masses which are unexplained by standard single star theory.

Iben has constructed linear series of helium shell burning models which could result from binary dwarf coalescence and identified a "super horizontal branch" where tracks converge in the $\mathrm{H}-\mathrm{R}$ diagram. This feature intersects the location of $\mathrm{R}$ CrB stars and hot helium stars given a plausible adjustment in their absolute magnitudes. This may provide evidence that $\mathrm{R}$ CrB stars result from the merger of $C O$ and He dwarfs.

Finally, Iben discussed problems involved in the formation and subsequent common envelope evolution of thermally pulsing AGB stars undergoing Case $C$ mass exchange. He noted that the common envelope could lead to friction and the reduction of the separation of the two cores, but that ejection of the envelope by the released gravitational energy of the settling cores was problematical. The basic planetary nebula ejection mechanism may play a role.

Barkat of Hebrew University discussed an important issue in the process of degenerate carbon ignition and thermonuclear explosion, the role of the convective Urca process. Degenerate carbon burning leads to thermonuclear runaway when the burning rate exceeds the neutrino loss rate. The runaway burning generates a grow- 
ing convective core, but the situation changes when the convective core extends beyond a critical density where the Fermi energy corresponds to the threshold for electron capture for a particular constituent. The convection then circulates mother and daughter nuclei past the critical threshold, so that alternate electron capture and decay occur with no net change in composition but the loss of a neutrino or anti-neutrino on each half of the cycle. This cyclic process is thus a copious source of cooling neutrinos in principle. The net effect has been uncertain, however, because the capture leaves a hole in the Fermi sea to be filled by a cascading electron and the decay deposits an electron with finite kinetic energy and thus the process also is a potential source of heating. Barkat argued that the convection will rapidly produce a steady state in which there is no net change in composition with time, but a gradient in composition of mothers, daughters and electrons, not a uniform composition. The currents in these species implied by the gradient $\overline{c a n}$ be shown to exactly balance the heating terms so that in steady state the heating is identically zero and the convective urca process is a strong source of net cooling as originally proposed by Paczynski.

Barkat further argued that the convective Urca cooling is unstable. The cooling is a very steep function of temperature since the process depends so sensitively on the extension of the convective core beyond the Urca shell at the critical threshold density. A positive perturbation in the core entropy will lead to much stronger neutrino losses and regulation. A negative perturbation, however, will lead to a smaller convective core and Urca losses reduced to balance the decreased nuclear energy production. Such a state will be susceptible to negative entropy perturbations due to ordinary neutrino losses and the only stable state is one in which the Urca process shuts off both itself and the thermonuclear burning to the point where the latter is balanced by the ordinary neutrino losses.

This process of central carbon ignition, thermal runaway, growing convective core, initiation of convective Urca cooling, and shutting down the burning by cooling the core will happen quickly in the core. The resulting configuration will have a strong step function downward in the inner temperature distribution in the convective core bounded by the Urca shell. The subsequent evolution will be dictated by the slow growth of the core mass and central density due to accretion. Further modeling should show whether the result is collapse if the central density reaches the critical threshold for dynamical electron capture/general relativistic instability, or explosion due to off-center carbon ignition in the hotter material which falls just beyond the urca shell.

\section{August 1988}

\section{General Scientific Session}

Chaired by A. Maeder
A.N. $\operatorname{Cox}$
Solar structure, oscillations and Neutrinos
G. Michaud
$\mathrm{Li}$ Abundance as a Constraint on Particle Transport
J. Truxan
Non-Spherical Effects: Case Study of Novae
M. Kato, I. Hachisu, and H. Saio
Mass Loss during Nova and Helium-Nova Outbursts
S. Sofia, M. Pinsonneault, S. Kawaler, and R. Demarque New Results on the Evolution of the Rotating Sun
C. Chiosi NGC 1866, an Observational Test for Convective Overshooting

This meeting was essentially devoted to hydrodynamical effects intervening in the course of stellar evolution.

Cox of Los Alamos National Laboratory constructed two precision solar models with the Iben evolution program; one with no-diffusion of the internal atomic 
nuclei, and the other with the effects of gravitational settling, thermal diffusion, and concentration gradient due to diffusion. Equation of state and opacity were fitted to the latest theoretical data. Then the opacity at the bottom of convection zone was revised to increase by 15 - 20 percent which allowed a better agreement with the observed solar p-mode frequencies. His theoretical p-mode frequencies are now lower only by a few microhertz than those observed. The helium concentrations of the initial mixture were $Y=0.291$ and 0.289 for the no-diffusion and diffusion models, respectively. The diffusion model evolved to a surface helium concentration of $\mathrm{Y}=0.256$ at the solar age, and the heavy element concentration, which was initially $z=0.0200$, decreased to $z=0.0179$ by diffusive settling. Calculation of g-mode solutions showed that they did not have equal period spacings until high radial order. Nonadiabatic solutions for these g-modes enabled him to predict their relative surface visibility. The high helium concentration which was necessitated by his equation of state, however, resulted in high central temperatures with 9 sNUs from the $B$ and 1.5 sNUs from the Be reactions. Neither a model with iron condensed-out deeper than the convection zone, nor a model with the presumed WIMPs to cool the central regions did reproduce observed p-mode separations.

Michaud of the University of Montreal gave an alternate explanation for the $L i$ abundance gap $($ at $T$, about $6700 \mathrm{~K}$ ) which is observed in clusters with ages between 4 and $20 \times 10^{8}$ years fof not in young clusters. According to usual understanding, the $T$, the width in $T$, and the maximum underabundance factor were thought as well effilined in terms 8 f gravitational settling at the age of Hyades. However, Boesgaard noticed that the $\mathrm{Li}$ abundance gap occurred where the equatorial rotational velocity increased rapidly with $T$, and she suggested that these two are related each other. Michaud and Effárbonneau expressed the envelope of observed rotational velocities in Hyades as a function of $T$. Then they calculated the transport of Li-free matter by meridional circulatiff from the region of temperature higher than $2.5 \times 10^{6} \mathrm{~K}$, and they were able to reproduce $\mathrm{Li}$ abundance gap with the observed width and depth.

Truran of the University of Illinois made a recall of the general properties of classical novae. In particular, various phases in evolution of classical novae were identified; accretion phase, thermonuclear runaway to maximum, post-maximum evolution in outburst, and return to minimum. Among many possible non-spherical effects, he discussed two of them. Firstly, as for the effects of magnetic field, he discussed the disruption of the accretion disk, the alteration of the accretion flow, the absence of shear mixing, and the possibility of polar thermonuclear ignition. Secondly, as for the common envelope evolution, he pointed out that it may result in observable effects on the visual light curve during the outburst.

Kato (Keio University) et al. followed evolution of shell flashes by a steady state approach describing it with stellar-wind mass-loss solutions. The mass loss rate was obtained uniquely: It is high while the star has extended envelope but it decreases as photospheric radius shrinks. The wind mass-loss ceases when the envelope mass reduces to a critical value. The time-dependent models were also calculated in helium shell flashes. The mass loss due to the Roche lobe overflow was taken into account. The mass accumulation ratio, i.e., the ratio of the mass remaining on the white dwarf after nova explosion to the mass having accumulated during the preceding accretion phase, was also calculated. It is less than about $1 / 3$ for high accretion rate and much less than 0.1 for low accretion rate for the case of hydrogen-flash nova. The Roche lobe overflow reduces this value. In the case of heliym-flash nova this ratio is less than 0.5 for the accretion rate lower

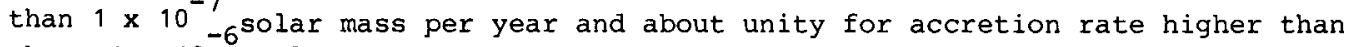
about $1 \times 10^{-6}$ solar mass per year.

Sofia et al. of Yale University developed a new code for evolution of rotating stars and applied it to the sun. Although the basic philosophy of Endal and Sofia (1976, 1978, 1981) was followed in the initial conditions and transport of angular 
momentum, the coding was totally independent and many details were reformulated: Accounts were taken for angular momentum loss via a magnetic wind and for angular momentum redistribution by rotationally induced instabilities. The resulting models have an oblateness in agreement with observed upper limits. The rotation curves of the models of the present-day sun must show the following features: The layers outer than 0.6 solar radius exhibit minimal differential rotation, while the central core inner than 0.2 solar radius preserves its initial rapid rotation. These basic features persist through a wide range of model parameters. Transport of angular momentum leads to rotationally induced mixing which explains the observed $L i$ depletion in the sun. The rotation curves in their models of the present-day sun are in qualitative agreement with the estimates from current analysis of solar oscillation for the layers outer than 0.6 solar radius.

Chiosi of Padova University studied the color-magnitude (CM) diagram and the main sequence luminosity function of the young IMC globular cluster NGC 1866 with the aim at discriminating among possible scenarios for evolution of intermediate mass stars. To this purpose, Johnson $B$ and $V$ CCD photometry of 1517 stars was obtained in the central region of NGC 1866, and of 640 stars in the nearby field. The CM diagram of this region was corrected for photometric incompleteness and for contamination with field stars. Comparison with the observational integrated luminosity function clearly demonstrated that models with convective overshoot ought to be preferred. In other words, the star counts in the cluster suggested that the ratio of core $\mathrm{H}$ - to He-burning lifetimes and the range of luminosity spanned by main sequence stars must be lower and wider, respectively, than those given by classical computations without the convective overshoot. This is possible only if such efficient mixing processes with convective overshoot really take place not only in the latest stages but also over all the preceding stages of the stellar evolution. It should be noticed that only such known mixing processes as semi-convection and/or breathing convection would not be able to satisfy the observational demand. 